\title{
Universal Ontogenetic Growth without Fitting Parameters: Implications for Life History Invariants \& Population Growth
}

\author{
Andrés Escala \\ Departamento de Astronomía, Universidad de Chile, Casilla 36-D, Santiago, Chile. \\ aescala@das.uchile.cl
}

\begin{abstract}
Since the work of Von Bertalanffy (1957), several models have been proposed that relate the ontogenetic scaling of energy assimilation and metabolism to growth, being able to describe ontogenetic growth trajectories for living organisms and collapse them onto a single universal curve (West et al. 2001; Barnavar et al. 2002). Nevertheless, all these ontogenetic growth models critically depends on fitting parameters and on the allometric scaling of the metabolic rate. Using a new metabolic rate relation (Escala 2019) applied to a Bertalanffy-type ontogenetic growth equation, we find that ontogenetic growth can also be described by an universal growth curve for all studied species, but without the aid of any fitting parameters. We find that the inverse of the heart frequency $\mathrm{f}_{\mathrm{H}}$, rescaled by the ratio of the specific energies for biomass creation and metabolism, defines the characteristic timescale for ontogenetic growth. Moreover, our model also predicts a generation time and lifespan that explains the origin of several 'Life History Invariants' (Charnov 1993) and predicts that the Mathusian Parameter should be inversely proportional to both the generation time and lifespan, in agreement with the data in the literature (Duncan et al. 1997; Dillingham et. al 2016; Hatton et al 2019). In our formalism, several critical timescales and rates (lifespan, generation time \& intrinsic population growth rate) are all proportional to the heart frequency $\mathrm{f}_{\mathrm{H}}$, thus their allometric scaling relations comes directly
\end{abstract}


from the allometry of the heart frequency, which is typically $\mathrm{f}_{\mathrm{H}} \propto M^{-0.25}$ under basal conditions.

\section{Introduction}

Metabolism and growth are two fundamental aspects of living organisms, thus is somewhat natural trying to understand the connections between both processes. Von Bertalanffy (1957) studied ontogenetic growth curves in order to establish connections between metabolism and growth, showing that individual growth curves in living organism can be reproduced from models of metabolic energy allocation. Although several subsequent growth models (Reiss 1989; West et al. 2001; Ricklefs 2003; Hou et al. 2008) differs significantly on the details of their derivation, it shares the same mathematical form of Von Bertalanffy (1957) that basically links patterns of assimilation and growth to the (allometric) scaling of metabolic rate. In particular, West et al. (2001) proposed a general quantitative model based on the allocation of metabolic energy and showed, that individual growth curves can be collapsed onto a single universal curve that describes the growth in all the studied species.

Nevertheless, Banavar et al (2002) illustrated later that the universal growth curve arises from general considerations on energy conservation that are independent of the specific allometric model used by West et al. (2001). In particular, Banavar et al (2002) showed that the data do not distinguish between the exact exponent in the scaling relationship between metabolic rate and mass, with both $2 / 3$ and $3 / 4$ exponents in the metabolic relation fitting equally well. This scaling collapse onto a universal curve that happens when some dimensionless quantities are properly defined, is also equivalent to asseverate that a single self-similar solution is able to successfully fit all the ontogenetic growth curves. Since the universal curve arises from general considerations, it is desirable any independent test on the assumptions behind the models that defines the key dimensionless quantities, which can be discerning between the different models for ontogenetic growth. 
In general, all ontogenetic growth models critically depends on the metabolic rate, specifically, on the allometric scaling which exact slope is still matter of debate (White et al. 2007). Recently, the empirical metabolic rate relation was corrected in order to fulfill dimensional homogeneity (Escala 2019), a minimal requirement for any meaningful law of nature (Bridgman 1922), proposing a new metabolic rate (B) formula: $B=\epsilon(T) \eta_{\mathrm{O}_{2}} \mathrm{f}_{\mathrm{H}} \mathrm{M}$, where $\mathrm{M}$ is the body mass, $\mathrm{f}_{\mathrm{H}}$ is a (characteristic) heart frequency, $\eta_{\mathrm{O}_{2}}$ is an specific $\mathrm{O}_{2}$ absorption factor for different exercising conditions (basal, maximal, etc) and $\epsilon(\mathrm{T})=\epsilon_{0} \mathrm{e}^{-\mathrm{E}_{\mathrm{a}} / \mathrm{kT}}$ is a temperature correction inspired in the Arrhenius formula (Gillooly et al. 2001), in which $\mathrm{E}_{\mathrm{a}}$ is an activation energy and $\mathrm{k}$ is the Boltzmann universal constant. Compared to Kleiber's original formulation (Kleiber 1932), $\mathrm{B}=\mathrm{B}_{0}\left(\mathrm{M} / \mathrm{M}_{0}\right)^{0.75}$, or the Rubner's surface rule proportional to $2 / 3$, this new metabolic rate relation has the heart frequency $\mathrm{f}_{\mathrm{H}}$ as controlling variable (a marker of metabolic rate) and the advantage of being an unique metabolic rate equation for different classes of animals and different exercising conditions, valid for both basal and maximal metabolic rates, in agreement with empirical data in the literature (Escala 2019). In addition, Escala (2020) showed that this new metabolic rate relation can be directly linked to the total energy consumed in a lifespan, being able to explain the origin of variations in the 'rate of living' theory (Speakman 2005; Ramsey et al. 2000).

In this paper, we explore the implications of this new metabolic relation for ontogenetic growth models, with a focus on independently test key quantities in our formulation that can be discerning with the previous models, being organized as follows. We start in $\S 2$ applying the results of the new metabolic rate relation (Escala 2019), to an ontogenetic growth equation that shares the same mathematical form of previous models, showing that individual growth curves can also be collapsed onto a single universal curve, but in this case without the aid of any fitting parameter. Section 3 continues with computing the predicted generation time and explaining the origin of several 'Life History Invariants', with satisfactory results. In $\S 4$, we study the predicted implications for population growth, showing that agrees with the collected data. Finally in $\S 5$, we discuss the results and final implications of this work. 


\section{Ontogenetic Growth Model}

Since different assumptions about the energy allocation get to the same general equation (von Bertalanffy 1957; Reiss 1989; West et al. 2001; Ricklefs 2003; Hou et al. 2008), we will follow the notation in the mass-energy conservation model described in Moses et al. (2008), that revisited the ontogenetic growth model of West et al. (2001), simply because we will compare to their results, using their parameters estimations. The conservation of energy for the allocation of metabolic energy during growth between maintenance of existing tissue and the production of new biomass, can be expressed as $E_{m} \frac{d m}{d t}=B-B_{m} m$ (Moses et al. 2008), where $\mathrm{B}$ is the metabolic rate (in $\mathrm{J} / \mathrm{s}$ or $\mathrm{W}), \mathrm{E}_{\mathrm{m}}$ (in $\mathrm{J} / \mathrm{g}$ ) is the energy required to create a unit of biomass and $\mathrm{B}_{\mathrm{m}}($ in $\mathrm{W} / \mathrm{g}$ ) is the metabolic rate required to maintain an existing unit of biomass.

For the corrected metabolic rate relation, we will restrict to basal (resting) conditions and neglect temperature variations, because ontogenetic growth happens over long periods of time where such variations might tend to cancel (in wild conditions). Under such conditions, is constant the factor $\epsilon(\mathrm{T}) \eta_{\mathrm{O}_{2}} \approx 10^{-4.313} \mathrm{mlO}_{2} \mathrm{~g}^{-1} \approx 10^{-3} \mathrm{~J} / \mathrm{g} \equiv \mathrm{E}_{2019}$ (converting 1 ltr $\mathrm{O}_{2}=20.1 \mathrm{~kJ}$; Schmidt-Nielsen 1984), where $\mathrm{E}_{2019}$ is a constant that comes from the best fitted value for the corrected metabolic relation (Eq. 8 of Escala 2019). Therefore, the metabolic rate formula is simply given by $\mathrm{B}=\mathrm{E}_{2019} \mathrm{f}_{\mathrm{H}} \mathrm{m}$ (Escala 2019) and assuming that the heart frequency scales with body mass $\mathrm{m}$ as $\mathrm{f}_{\mathrm{H}}=\mathrm{f}_{0} \mathrm{~m}^{-\alpha}$, (mass-)energy conservation can be expressed as

$$
\frac{\mathrm{dm}}{\mathrm{dt}}=\mathrm{am}^{1-\alpha}-\mathrm{bm}
$$

where $\mathrm{a}=\mathrm{E}_{2019} \mathrm{f}_{0} / \mathrm{E}_{\mathrm{m}}$ and $\mathrm{b}=\mathrm{B}_{\mathrm{m}} / \mathrm{E}_{\mathrm{m}}$. The general solution of $\mathrm{Eq} 1$ is a classical sigmoidal curve, which general form is given by von Bertalanffy (1957; Eq 6). Noting also that for an initial (birth) mass $m_{0}$ and final (asymptotic) mass $\mathrm{M}$, the condition $\mathrm{dm} / \mathrm{dt}=0$ (at $\mathrm{m}=$ 
$\mathrm{M})$ in $\mathrm{Eq} 1$ is equivalent to $\mathrm{a} / \mathrm{b}=\mathrm{M}^{\alpha}$, the solution to $\mathrm{Eq} 1$ can be written as:

$$
\left(\frac{\mathrm{m}}{\mathrm{M}}\right)^{\alpha}=1-\left[1-\left(\frac{\mathrm{m}_{0}}{\mathrm{M}}\right)^{\alpha}\right] \times \exp \left(-\frac{\mathrm{at}}{\alpha^{-1} \mathrm{M}^{\alpha}}\right)
$$

This solution is equivalent to the one found by Banavar et al. (2002) and for the special case of $\alpha=1 / 4$, gives the solution given in West et al (2001) (their Eq 5). The solution given by Eq 2 can be rewritten as $\mathrm{r}=1-\mathrm{e}^{-\tau}$, which is the same universal growth curve found in West et al (2001) (and Banavar et al 2002), but using in our case the following variable change:

$$
\mathrm{r} \equiv\left(\frac{\mathrm{m}}{\mathrm{M}}\right)^{\alpha} ; \tau \equiv \frac{\mathrm{E}_{2019}}{\mathrm{E}_{\mathrm{m}}} \frac{\mathrm{f}_{\mathrm{H}} \mathrm{t}}{\alpha^{-1}}-\ln \left[1-\left(\frac{\mathrm{m}_{0}}{\mathrm{M}}\right)^{\alpha}\right]
$$

where we also replaced $\mathrm{a}=\mathrm{E}_{2019} \mathrm{f}_{0} / \mathrm{E}_{\mathrm{m}}$ in $\mathrm{Eq} 2$ (in the definition of $\tau$ ) and thus, $\mathrm{f}_{\mathrm{H}}=\mathrm{f}_{0} \mathrm{M}^{-\alpha}$ is (from now) the heart frequency when the animal reaches the final (asymptotic) mass M. For the particular case of $\alpha=1 / 3, \mathrm{r}$ corresponds also to the fractional size $\left(\mathrm{r}=(\mathrm{m} / \mathrm{M})^{1 / 3}=l / \mathrm{L}\right)$, leading to the classical Bertalanffy growth equation, $l(\tau)=\mathrm{L}\left(1-\mathrm{e}^{-\tau}\right)$, which has been successfully applied in the fishery industry (Beverton \& Holt, 1959; Charnov 2008). In this case, we can explicitly express (in terms of physical quantities) the Bertalanffy growth coeficient as $\mathrm{K} \equiv \tau / \mathrm{t}=\frac{\mathrm{E}_{2019}}{\mathrm{E}_{\mathrm{m}}} \frac{\mathrm{f}_{\mathrm{H}}}{\alpha^{-1}}\left(\right.$ from $\mathrm{Eq} 3$ for $\left.\mathrm{m}_{0}=0\right)$.

The advantage of this formulation compared to previous ones, is that now the dimensionless time $\tau$ is expressed in terms of quantities that are well defined, with clear either physical $\left(\mathrm{E}_{2019}, \mathrm{E}_{\mathrm{m}}\right)$ or biological $\left(\mathrm{f}_{\mathrm{H}}, \mathrm{M}\right.$, etc) meaning, therefore, the dimensionless variables defined in Eq. 3 are now written in a physically transparent form, without the aid of fitting parameters (for example, the parameter a has a fractal dimensionality of $\left[\operatorname{mass}^{\alpha}\right] /[$ time $]$ and is critical in fitting the growth curves in Bertalanffy 1957, West et al 2001, Banavar et al 2002, etc). Noteworthy is that in this formulation for $\tau$, the inverse of the heart frequency $\mathrm{f}_{\mathrm{H}}^{-1}$ defines the characteristic timescale for ontogenetic growth, $\mathrm{t}_{\text {growth }} \equiv \mathrm{f}_{\mathrm{H}}^{-1} \frac{\mathrm{E}_{\mathrm{m}}}{\mathrm{E}_{2019}}$, being rescaled by the ratio of specific energies of biomass creation $\left(\mathrm{E}_{\mathrm{m}}\right)$ and (basal) metabolism $\left(\mathrm{E}_{2019}\right)$.

Banavar et al. (2002) showed that the ontogenic growth curves do not distinguish between an exponent of $2 / 3$ or $3 / 4(=1-\alpha)$ in the mass scaling of the metabolic rate 
relation, being both an equally good fit of the current data and consistent with an universal curve $\mathrm{r}=1-\mathrm{e}^{-\tau}$ for ontogenetic growth. Therefore, only for consistency we will assume $\alpha=1 / 4$, since we will compare our formulation against data using the fitting parameters from West et al (2001), which assumes such $\alpha$ value.

Since the universal growth curve $\mathrm{r}=1-\mathrm{e}^{-\tau}$, for the $\tau$ defined in Eq. 3 (with $\alpha=1 / 4$ ), is mathematically identical to the solution found in West et al (2001) (their Eq 5), when the following condition is fulfilled:

$$
\mathrm{t}_{\text {growth }}^{-1} \equiv \frac{\mathrm{E}_{2019}}{\mathrm{E}_{\mathrm{m}}} \mathrm{f}_{\mathrm{H}}=\frac{\mathrm{a}}{\mathrm{M}^{1 / 4}}
$$

then it should fit equally well the individual growth curves for the species studied in West et al $(2001)$, if their heart frequencies $\mathrm{f}_{\mathrm{H}}$ and specific energies $\left(\mathrm{E}_{2019}, \mathrm{E}_{\mathrm{m}}\right)$ fulfill the condition given by $\mathrm{Eq} 4$, for the parameter a and final mass $\mathrm{M}$ found in the fitting procedure of the growth curves in West et al (2001). In order to test if the condition in Eq. 4 is fulfilled in nature, we collected estimations for the energy required to create a unit of biomass $\mathrm{E}_{\mathrm{m}}$ (Moses et al. 2008, taking averages when multiple estimations are available) and measurements of heart frequencies $\mathrm{f}_{\mathrm{H}}$ for the species studied in West et al (2001), in order to complement them with the parameters a, birth mass $\mathrm{m}_{0}$ and final mass $\mathrm{M}$ that best fit the growth curves studied in West et al (2001). A summary of all the quantities (with the individual references for $\mathrm{f}_{\mathrm{H}}$ ) are listed in Table 1.

Table 1 


\begin{tabular}{||cccccc||}
\hline Species & $\mathrm{a}\left[\mathrm{g}^{1 / 4} / \mathrm{day}\right]$ & $\begin{array}{c}\mathrm{m}_{0}[\mathrm{~kg}] \\
\text { West et al } 2001\end{array}$ & $\mathrm{M}[\mathrm{kg}]$ & $\begin{array}{c}\mathrm{E}_{m}[\mathrm{~J} / \mathrm{g}] \\
\text { Moses et al. 2008 }\end{array}$ & $\begin{array}{c}\mathrm{f}_{H}[\# / \mathrm{min}] \\
\text { (Reference) }\end{array}$ \\
\hline \hline Cow & 0.28 & 33.3 & 442 & 6950 & 63 (Kovacs et al 2005) \\
\hline Pig & 0.31 & 0.90 & 320 & 6150 & 70 (Harris 2009) \\
\hline Rabbit & 0.36 & 0.12 & 1.35 & 6100 & 180 (Detweiler et al. 2004) \\
\hline Guinea pig & 0.21 & 0.005 & 0.840 & 4900 & 277 (Weibel et al. 2004) \\
\hline Rat & 0.23 & 0.008 & 0.280 & 4350 & 393 (Weibel et al. 2004) \\
\hline Shrew & 0.83 & 0.0003 & 0.0042 & 1800 & 835 (Jurgens et al. 1996) \\
\hline Heron & 1.56 & 0.003 & 2.7 & 1400 & 211 (Machida et al. 2001) \\
\hline Robin & 1.9 & 0.001 & 0.022 & 2000 & 570 (Welty et al.1988) \\
\hline Cod & 0.017 & 0.0001 & 25 & 13000 & 32 (Wardle et al. 1973) \\
\hline Salmon & 0.026 & 0.00001 & 2.4 & 7300 & 30 (Clark et al. 2011) \\
\hline Guppy & 0.10 & 0.000008 & 0.00015 & 1900 & 120 (Unpublished data) \\
\hline
\end{tabular}

Figure 1 display the relation given by Eq 4, between the variables a, $\mathrm{M}, \mathrm{E}_{\mathrm{m}} \& \mathrm{f}_{\mathrm{H}}$ as a function of the final body mass M. Besides the 7 orders of magnitude in body mass $M$ variations, the relation has a slope consistent with zero $\left(\propto \mathrm{M}^{-0.05}\right.$ and with only 0.22 dex in scatter, comparable to the scatter in metabolic rate relation found in Escala 2019), confirming that the condition in Eq. 4 is fulfilled. This implies that for the specific dimensionless variables defined in Eq. 3, the universal curve $\left(\mathrm{r}=1-\mathrm{e}^{-\tau}\right)$ will describe the (different) individual growth curves as well as the models in West et al (2001) (and Banavar et al. 2002).

Moreover, the different species displayed in Figure 1 are scattered around the predicted value of $\mathrm{E}_{2019}\left(=10^{-3} \mathrm{Jg}^{-1}\right.$; Escala 2019) denoted by the red dashed line, therefore, Fig 1 can be considered the third independent estimation of $\mathrm{E}_{2019}$ (in addition of Escala 2019, 2020), supporting the predictability of the formalism presented and showing something characteristic of the exact sciences: fewer (and universal) constants having consistent 


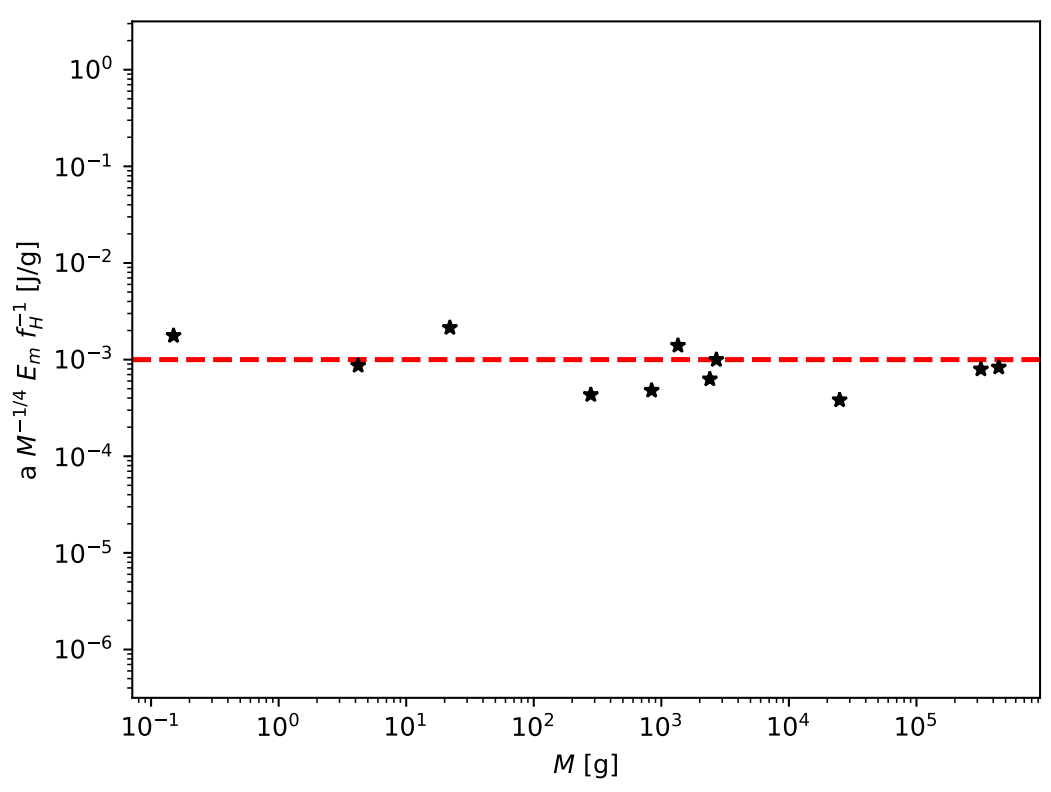

Fig. 1. - The relation between the variables a, $M, \mathrm{E}_{\mathrm{m}} \& \mathrm{f}_{\mathrm{H}}$, as a function of the final body mass M, showing that does not vary systematically with M, as predicted by Eq 4 . The different species displayed in black stars are scattered around the predicted value of $\mathrm{E}_{2019}$, which is denoted by the red dashed line. The best-fitted slope is consistent with zero, $\frac{\mathrm{a}}{\mathrm{M}^{1 / 4}} \frac{\mathrm{E}_{\mathrm{m}}}{\mathrm{f}_{\mathrm{H}}} \propto \mathrm{M}^{-0.05}$, with only 0.22 dex in scatter.

measurements in multiple contexts. Finally, Figure 1 strongly supports that the inverse of the heart frequency $\mathrm{f}_{\mathrm{H}}^{-1}$, rescaled by the ratio of the competing specific energies (biomass creation $\mathrm{E}_{\mathrm{m}}$ versus metabolism $\mathrm{E}_{2019}$ ), defines the characteristic timescale for ontogenetic growth $\mathrm{t}_{\text {growth }}=\mathrm{f}_{\mathrm{H}}^{-1} \frac{\mathrm{E}_{\mathrm{m}}}{\mathrm{E}_{2019}}$.

\section{Generation-Time and the origin of some Life History Invariants}

The success of the formulation given by Eq. 3, supported by Fig 1, motivates us to study its implications and for that reason, it is interesting to look the predicted generation-time 
$t_{\text {gen }}$, which is the time period required for young to grow to its final size and thus mature to reproductive age. The generation time $t_{\text {gen }}$ can be straightforwardly determined from the $\tau$ defined in Eq. 3, being possible to arrive to the following relation

$$
\mathrm{t}_{\text {gen }}=\left(\tau^{*}+\ln \left[1-\left(\frac{\mathrm{m}_{0}}{\mathrm{M}}\right)^{\alpha}\right]\right) \frac{\mathrm{E}_{\mathrm{m}}}{\mathrm{E}_{2019}} \frac{\alpha^{-1}}{\mathrm{f}_{\mathrm{H}}} \propto \mathrm{f}_{\mathrm{H}}^{-1}
$$

where $\tau^{*}$ is the approximate dimensionless time value to reach adult mass (for all species) in the universal growth curve. Eq. 5 gives a generation-time explicitly dependent on the heart frequency $\mathrm{f}_{\mathrm{H}}$, where for the allometric scaling of the heart frequency under basal conditions $\left(\mathrm{f}_{\mathrm{H}} \propto \mathrm{M}^{-0.25}\right.$; Brody 1945, Calder 1968) gives the well known mass scaling for the generationtime $\left(\mathrm{t}_{\text {gen }} \propto \mathrm{M}^{0.25}\right.$; Bonner 1965). Nevertheless, since most biological rates and times scale as $\mathrm{M}^{-1 / 4}$ and $\mathrm{M}^{1 / 4}$ (Savage et al. 2004, Burger et al. 2021), an interesting possible test is to study allometric variations of $t_{\text {gen }}$ in big outliers from ' $1 / 4$ scaling' such as spiders (Anderson 1970, 1974) and other organisms (White et al. 2007), testing if they vary like $\mathrm{f}_{\mathrm{H}}$ as predicted by Eq. 5. Another interesting possibility is to directly test the predicted inverse correlation between $t_{\text {gen }}$ and $f_{H}$.

One of the advantages of having a generation time explicitly dependent on the heart frequency $\mathrm{f}_{\mathrm{H}}$, is that it can be directly linked (Escala 2019, 2020) to the total lifespan $\mathrm{t}_{\text {life }}$, using the empirical relation of total number of heartbeats $\left(\mathrm{N}_{\mathrm{b}}\right)$ in a lifetime $t_{\text {life }}=\mathrm{N}_{\mathrm{b}} / \mathrm{f}_{\mathrm{H}}$ found to be valid in mammals (Levine 1997; Cook et. al 2006). Escala (2020) generalized this relation for all type of living organisms, using the proportionally between heart and respiration frequencies, $\mathrm{f}_{\mathrm{H}}=\mathrm{kf}_{\text {resp }}$ (Schmidt-Nielsen 1984), then the empirical relation with lifetime can be rewritten to be valid also for a total number $\mathrm{N}_{\mathrm{r}}\left(=\mathrm{N}_{\mathrm{b}} / \mathrm{k}\right)$ of 'respiration cycles': $\mathrm{t}_{\text {life }}=\mathrm{N}_{\mathrm{b}} / \mathrm{f}_{\mathrm{H}}=\mathrm{N}_{\mathrm{b}} / \mathrm{kf}_{\text {resp }}=\mathrm{N}_{\mathrm{r}} / \mathrm{f}_{\text {resp }}$. Escala (2020) also used this relation to predict the total lifespan energy consumed in living organisms, with satisfactory results if it is required an approximately constant total number $\mathrm{N}_{\mathrm{r}} \sim 10^{8}$ of respiration cycles per lifetime in all living organisms, which further supports the generalization. Combining this relation for a fixed number of respiration cycles per lifetime with Eq 5, we arrive to:

$$
\mathrm{G} \equiv \frac{\mathrm{t}_{\text {gen }}}{\mathrm{t}_{\text {life }}}=\left(\tau^{*}+\ln \left[1-\left(\frac{\mathrm{m}_{0}}{\mathrm{M}}\right)^{\alpha}\right]\right) \frac{\mathrm{E}_{\mathrm{m}} \alpha^{-1}}{\mathrm{E}_{2019} \mathrm{~N}_{\mathrm{b}}} \approx \frac{\mathrm{E}_{\mathrm{m}}}{\mathrm{k}} \frac{4 \tau^{*}}{\mathrm{E}_{2019} \mathrm{~N}_{\mathrm{r}}},
$$


where we also approximate for $\mathrm{m}_{0}<<\mathrm{M}$ (in agreement with the species listed in Table 1), neglecting minor changes due to the weakly varying logarithm. Eq. 6 directly relates the generation time and lifespan to their respective energies (per unit mass) to create biomass $\left(\mathrm{E}_{\mathrm{m}}\right)$ and sustain lifespan $\left(\mathrm{N}_{\mathrm{b}} \mathrm{E}_{2019}\right)$, only by assuming (mass-)energy conservation (Eq. 1) and with the invariant $\mathrm{N}_{\mathrm{r}}\left(=1.6210^{8}\right.$ respiration cycles per lifetime; Escala 2020) as critical link between both timescales.

Eq. 6 can be directly compared to the data compiled by Charnov \& Berrigan (1990), that summarizes published data for the ratio $t_{\text {adult }} / t_{\text {gen }}$ in different animal groups, where $\mathrm{t}_{\mathrm{adult}}=\mathrm{t}_{\text {life }}-\mathrm{t}_{\text {gen }}$ is the adult lifespan and noting that such definition is related to the $\mathrm{G}$ ratio as $\mathrm{t}_{\text {gen }} / \mathrm{t}_{\text {life }}=\left(1+\mathrm{t}_{\text {adult }} / \mathrm{t}_{\text {gen }}\right)^{-1}$. Also, noting that in Charnov \& Berrigan (1990) lifespans are estimated from the inverse of (instantaneous) mortality rates, being effectively fields lifespans and not maximum ones, thus to compare with Eq. 6 a correcting factor 2.5 must be included (McCoy \& Gillooly 2008), namely $\mathrm{G}^{\min }=\mathrm{t}_{\text {gen }} / \mathrm{t}_{\text {life }}^{\max }=\left(2.5\left(1+\mathrm{t}_{\text {adult }} / \mathrm{t}_{\text {gen }}\right)\right)^{-1}$.

Table 2 summarizes the estimations of $\mathrm{G}^{\text {min }}$ using the data compiled in Charnov \& Berrigan (1990) and compared it with the predicted values from Eq. 6, using estimates of $\mathrm{E}_{\mathrm{m}}$ for taxonomic groups (average of juvenile estimates; Moses et al. 2008), $\mathrm{k}$ values for such groups (Schmidt-Nielsen 1984; Escala 2020) and a dimensionless time $\tau^{*} \sim 5$, the approximate value to reach adult mass in universal growth curve (i.e. flat part of the curve in Fig. 2 of West et al 2001). We find that the predicted values are overall consistent with the empirically determined (Table 2), but taking into account that each taxonomic groups of birds, mammals and fishes includes species with up to an order of magnitude different $\mathrm{E}_{\mathrm{m}}$ (i.e. cod vs guppy fish in Table 1) and not necessarily the same species studied in Charnov \& Berrigan (1990), what is even more relevant is that the relative $\mathrm{G}^{\text {min }}$ trends (between birds, mammals and fish) observed in Charnov \& Berrigan (1990) are successfully predicted by the ratio $\frac{\mathrm{E}_{\mathrm{m}}}{\mathrm{k}}$.

Table 2 


\begin{tabular}{||ccccc||}
\hline Animal Group & $\mathrm{E}_{m}[\mathrm{~J} / \mathrm{g}]$ & $\mathrm{k}$ & Predicted Eq 6 & $\mathrm{G}^{\min }=\frac{\mathrm{t}_{\mathrm{gen}}}{\mathrm{t}_{\mathrm{life}}^{\max }}$ \\
\hline \hline Birds & 4050 & 9 & 0.06 & 0.11 \\
\hline Mammals & 5800 & 4.5 & 0.16 & 0.17 \\
\hline Fish & 7400 & 3 & 0.3 & 0.28 \\
\hline
\end{tabular}

Eq. 6 shows that the invariant relation between lifespan and age at maturity that is valid within taxonomic groups, as observed by Charnov \& Berrigan (1990), might come directly from the existence of another invariant: the approximately constant total number $\mathrm{N}_{\mathrm{r}} \sim 10^{8}$ of respiration cycles per lifetime. This $t_{\text {adult }} / \mathrm{t}_{\text {gen }}$ ratio is indeed one of the studied dimensionless life-history invariants (Calder 1984; Charnov 1993), which sometimes has been criticized to be spurious, just being a form of 'regressing $\mathrm{X}$ on $\mathrm{X}$ ' a random number (Nee et al. 2005). In this work, the generation and lifespan timescales are quantities derived independently, giving a quantitative prediction for being approximately invariant ratio within taxonomic groups, including their inter-specific variations, that comes from the basics energetics of respiration and the creation of new biomass and therefore, having a clear physical interpretation and thus far from being spurious.

Traditionally this relation, expressed in terms of the adult lifespan and age at maturity (which typically marks the stop of animals growth), as in Charnov \& Berrigan (1990), has been qualitative explained in terms of life-history evolution theory. Many versions lifehistory theories predict that the age of maturity should be positively correlated with the lifespan (Charnov 1993), being these patterns a reflection of natural selection (Charnov \& Berrigan 1991). In this paper, after rewriting the properties of living organisms in a physical transparent form, we predict the value of this life-history invariant in terms of the relevant energetics $\left(\mathrm{E}_{\mathrm{m}}, \mathrm{E}_{2019}, \mathrm{k}\right.$, etc) and find that the constancy mainly comes from the invariant number $\mathrm{N}_{\mathrm{r}} \sim 10^{8}$ of respiration cycles per lifetime, a generalization of the well known relation of constant number of heartbeats in mammal's lifetime (Levine 1997; Cook et. al 2006). Nevertheless, we do not study the origin of how the key parameters $\left(\mathrm{E}_{\mathrm{m}}, \mathrm{k}\right.$, etc) varies across species, animal groups and generations, which should be evolutionary in 
origin and thus set by natural selection (Charnov 1993; Gardner et al 2005).

It is direct to realize that some of the other life-history invariants (Charnov 1993), comes directly from the existence of invariant $\mathrm{G}=\mathrm{t}_{\mathrm{adult}} / \mathrm{t}_{\mathrm{gen}}$ and the universal ontogenetic growth curve. For example, the invariant ratio between the mortality rate (M) and the Bertalanffy growth coefficient (K) (Beverton \& Holt 1963, Cushing 1968, Pauly 1980), comes from its the relation with $\tau$ in our formulation, $\mathrm{Kt}=\tau$, which at the generation time corresponds to $\mathrm{Kt}_{\text {gen }}=\tau^{*} \sim 5$, according to the universal growth curve (West et al. 2001; Barnavar et al. 2002). Noting also that the mortality rate $\mathrm{M}$ is approximately the inverse of the lifespan $1 / \mathrm{t}_{\text {life }}$ (in the next section we will see a more rigorous definition for $\mathrm{M}$ ), is straightforward to find that $\mathrm{M} / \mathrm{K}=\mathrm{t}_{\text {gen }} /\left(\mathrm{t}_{\text {life }} \tau^{*}\right)=\mathrm{G} / 5$. Another invariant is the ratio between the length at maturity $l\left(\mathrm{t}_{\text {gen }}\right)$ and maximum asymptotic length $\mathrm{L}$ (Charnov \& Berrigan 1991), which comes also from $\mathrm{Kt}_{\text {gen }}=\tau^{*} \sim 5$, simply recalling the Bertalanffy growth equation (derived in $\S 2): l\left(\mathrm{t}_{\text {gen }}\right) / \mathrm{L}=1-\mathrm{e}^{-\tau^{*}}$. Finally, the invariant fraction of body mass to reproduction per unit time per life span (Charnov, Turner \& Winemiller 2001), comes from the definition of the dimensionless mass $\mathrm{r}(\mathrm{Eq} 3)$ and the invariant $\mathrm{G}(\mathrm{Eq} 6)$, being related as $\frac{\mathrm{m}\left(\mathrm{t}_{\text {gen }}\right)}{\mathrm{M}} \frac{\mathrm{t}_{\text {life }}}{\mathrm{t}_{\text {gen }}}=\left(1-\mathrm{e}^{-\tau^{*}}\right)^{1 / \alpha} \mathrm{G}^{-1}$, noting again that $\tau^{*} \sim 5$.

\section{Implications for Population Growth: Mathusian Parameter and the 'Equally Fitness Paradigm'}

Mathus (1798) studied the simplest model of population growth, which can be derived assuming that all individuals are identical and reproduce continuously, therefore, the number of individuals $\mathrm{N}$ will change with $\mathrm{B}$ births rates and $\mathrm{D}$ deaths rate as follows:

$$
\frac{\mathrm{dN}}{\mathrm{dt}}=\mathrm{B}-\mathrm{D}=\left(\mathrm{b}_{\mathrm{N}}-\mathrm{d}_{\mathrm{N}}\right) \mathrm{N}=\mathrm{r}_{\mathrm{m}} \mathrm{N}
$$

where $b_{N}$ and $d_{N}$ are per capita birth and death rates respectively, being $r_{m}$ the Malthusian parameter or intrinsic (maximum) population growth rate. The solution of Eq. 7 has an exponential form, given by $\mathrm{N}(\mathrm{t})=\mathrm{N}_{0} \mathrm{e}^{\mathrm{r}_{\mathrm{m}} \mathrm{t}}$ and therefore, the Malthusian parameter 
$\mathrm{r}_{\mathrm{m}}=\mathrm{b}_{\mathrm{N}}-\mathrm{d}_{\mathrm{N}}$ has units of inverse time and can be rewritten in the following form: $\mathrm{r}_{\mathrm{m}}=$ $\overline{\mathrm{b}} / \mathrm{t}_{\text {adult }}-\overline{\mathrm{d}} / \mathrm{t}_{\text {life }}$. The constants $(\overline{\mathrm{b}}, \overline{\mathrm{d}})$ are now dimensionless, since we have identified $\mathrm{t}_{\text {life }}$ as the characteristic timescale for death rates and $t_{\text {adult }}=t_{\text {life }}-t_{\text {gen }}$, as the characteristic timescale for the birth rates. The reason for the latter, is that $t_{\text {adult }}$ is the reproductive adult lifespan, where it is subtracted from the lifespan the time period required to (grow and) mature to reproductive age, $\mathrm{t}_{\mathrm{gen}}$, a time period where is not fulfilled a key assumption in Eq 7 , that the individuals are able to reproduce (continuously).

Using the Eq 6 that relates $t_{\text {gen }}$ and $t_{\text {life }}$, the Malthusian parameter is then given by:

$$
\mathrm{r}_{\mathrm{m}}=\left(\frac{\overline{\mathrm{b}}}{1-\mathrm{G}}-\overline{\mathrm{d}}\right) \mathrm{t}_{\text {life }}^{-1}=\left(\frac{\overline{\mathrm{b}}}{1-\mathrm{G}}-\overline{\mathrm{d}}\right) \frac{\mathrm{f}_{\mathrm{H}}}{\mathrm{N}_{\mathrm{b}}} \propto \mathrm{f}_{\mathrm{H}}
$$

which gives a Malthusian parameter $r_{m}$ again explicitly dependent on the heart frequency $\mathrm{f}_{\mathrm{H}}$. For the allometric scaling of heart frequency under basal conditions $\left(\mathrm{f}_{\mathrm{H}} \propto \mathrm{M}^{-0.25}\right.$, Brody 1945, Calder 1968) gives the well known Fenchel (1974) allometry, $\mathrm{r}_{\mathrm{m}} \propto \mathrm{M}^{-0.25}$, but again as most biological rates and times scale as $\mathrm{M}^{-1 / 4}$ and $\mathrm{M}^{1 / 4}$ (Savage et al. 2004, Burger et al. 2021), a more interesting test will be to study allometric variations of $r_{m}$ in outliers from ' $1 / 4$ scaling' in the heart frequency $f_{H}$, testing if they vary like $f_{H}$ as predicted by Eq. 8 .

Another prediction of Eq. 8 is an inverse correlation between $r_{m}$ and $t_{\text {life, }}$ which was recently tested by Hatton et al (2019). Fig 2 display the data of Hatton et al (2019) for all types of living organisms, ranging from protists to mammals and birds. Since the present analysis is restricted to organisms with heart, we denoted with the dashed (blue) line the mass scale of $10^{-3}$ grams, which corresponds to the scale associated to smallest organisms with heart, such as fruit or fairly flies, which coincides in Fig 2 with a transition to a decrease in scatter (for larger masses) and in addition, all individual animal groups (colors in Fig 2) fulfill the relation for masses larger that $10^{-3}$ grams. The solid black line corresponds to the best fit to the data, consistent with a clear inverse correlation between $r_{m}$ and $t_{\text {life, }}$ with no residual slope with body mass $\left(\mathrm{r}_{\mathrm{m}} \mathrm{t}_{\text {life }}=3.35 \mathrm{M}^{0.01}\right.$, scatter of $\left.0.5 \mathrm{dex}\right)$.

The relative low variation (on average one order of magnitude since the scatter is 0.5 


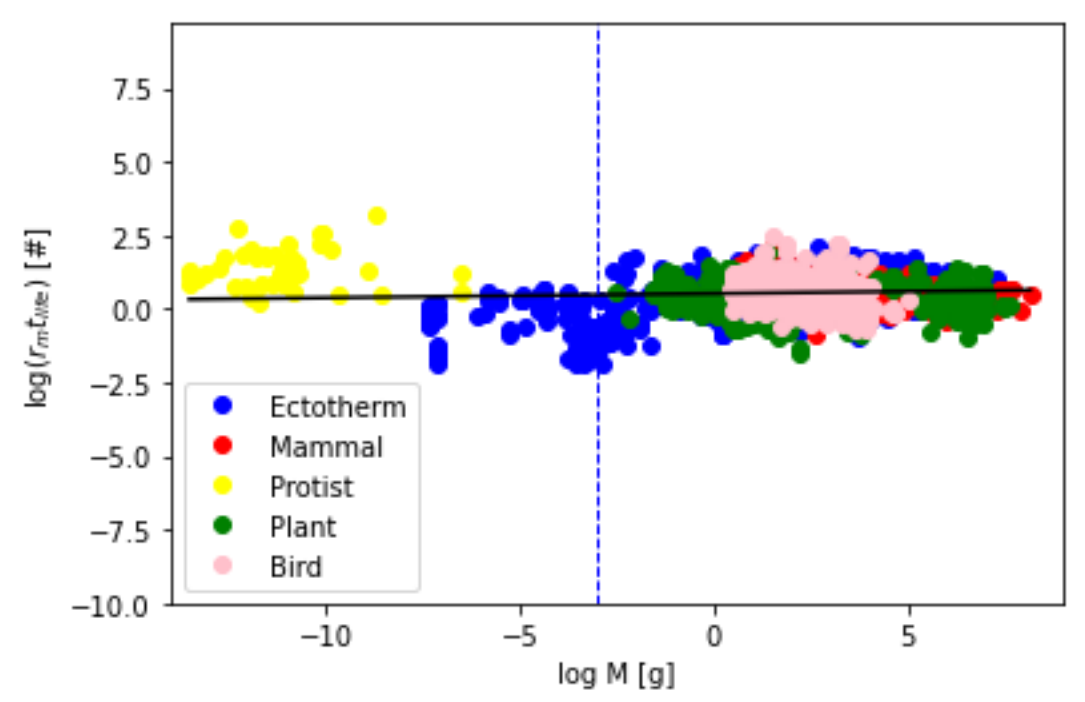

Fig. 2.- Lifetime Growth: Intrinsic (maximum) population growth rates $\left(\mathrm{r}_{\mathrm{m}}\right)$ multiplied by their lifespans $\left(\mathrm{t}_{\text {life }}\right)$ for all types of living organisms, ranging from protists to mammals and birds, compiled by Hatton et al (2019). The solid black line corresponds to the best fit to the data, with no residual slope with body mass, $\mathrm{r}_{\mathrm{m}} \mathrm{t}_{\text {life }}=3.35 \mathrm{M}^{0.01}$, with a scatter of 0.5 dex. The dashed blue line the mass scale of $10^{-3}$ grams, which corresponds to the scale associated to the smallest organisms with heart, where the present analysis is restricted.

dex) and specially, the negligible slope with body mass showed in Fig 2 has been claimed (Hatton et al 2019) to support the so-called 'Equally Fitness Paradigm' (Brown et al 2018; Burger et al. 2020), which states that "most organisms are more or less equally fit, as evidenced by the persistence of millions of plant, animal and microbe species of widely varying size, form and function in the Earth's diverse environments" (Brown et al 2018). In terms of the proposed formalism, Eq. 8 and the low variation of $\mathrm{G}$ across animals groups (the factors 2-3 seen in Table 2), implies also a low variation for the parameters $\overline{\mathrm{b}}$ and $\overline{\mathrm{d}}$ across species. These relatively constant values (implied by Fig 2) for the dimensionless parameters $(\bar{b}, \bar{d})$ associated to the (net) per capita birth and death per characteristic time, besides the spectacular diversity of life histories in terms growth, reproduction and survivorship over the life cycle, which implies very diverse life history strategies in the huge mass range displayed 
in Fig 2, supports that selection mechanisms should operate, in terms of equal fit according to Darwinian evolution.

In the fundamental theorem of natural selection, Fisher (1930) states that the rate of increase of mean fitness (caused by natural selection; Price 1972) is equal to the genetic variance of a species, conceptually linking natural selection with genetics. Natural selection can only increase fitness by reducing genetic variance (i.e. selecting away undesirable alleles; Basener \& Sanford 2018), thus without mutations and given enough time, selection must reduce genetic variance all the way to zero and fitness must reach a maximum, according to Fisher's theorem and confirmed thru simulations (Basener \& Sanford 2018). In general, entropy of a given probability distribution depends on its variance, for example the Gaussian distribution maximizes the entropy for a given variance, thus to reduce genetic variance implies a reduction of genetic entropy, which according to the second law of thermodynamics must be compensated with an increase in entropy in another component of the system (in an isolated system total entropy cannot decrease), which in this case should be an increase in the fitness entropy. The 'Equally Fitness Paradigm' seems to suggest that a constant (maximum) fitness value has been reached by the coexisting species in the current (environment) conditions. Also, according to the second law of thermodynamics maximum entropy happens only in the state of thermodynamic equilibrium, where (the theorem of) equipartition of energy holds. The 'Energetic Equivalence Rule' (Damuth 1981), which has been recently verified for $\sim 3000$ species in Hatton et al. (2019), seem to be a version of such equipartition of energy in live matter and thus, also consistent with being in such state when maximum entropy is reached.

Another relation for $r_{m}$ that can be derived using the definition of $\mathrm{G}$ (Eq 6), is the one which predicts an inverse correlation with the generation time $t_{\text {gen }}$ :

$$
r_{m}=\left(\frac{G \bar{b}}{1-G}-G \bar{d}\right) \frac{1}{t_{\text {gen }}} \propto t_{\text {gen }}^{-1},
$$

a relation that again can be tested. For that purpose, we use now the data in Duncan et al. 
(1997) that compiles ages at first reproduction $\left(\sim \mathrm{t}_{\text {gen }}\right)$ and maximum population growth rates for mammals. Fig 3 displays the data collected in Duncan et al. (1997), which is in overall agreement with Eq 9, with no relevant slope with body mass. The solid line denotes the best-fit to the data $\left(\mathrm{r}_{\mathrm{m}} \mathrm{t}_{\text {gen }}=1.38 \mathrm{M}^{-0.067}\right)$, which has an average scatter of 0.2 dex. Dillingham et. al (2016) also found equivalent results on this expected inverse relationship, using more sophisticated bayesian analysis.

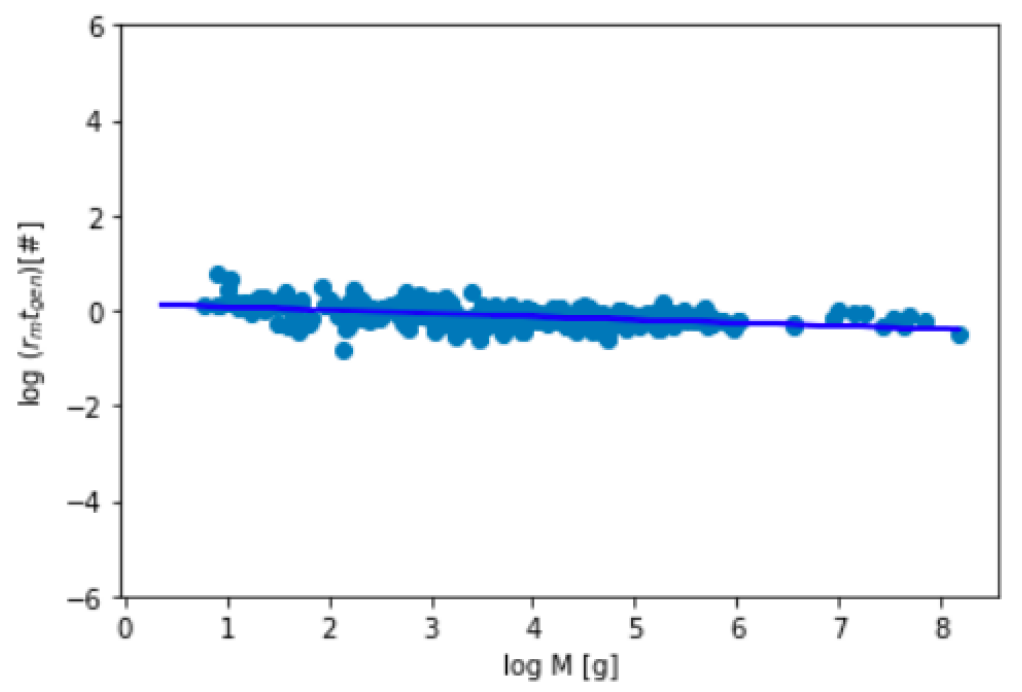

Fig. 3.- Generation times $\left(t_{\text {gen }}\right)$ times the maximum population growth rates $\left(\mathrm{r}_{\mathrm{m}}\right)$ for mammals, compiled by Duncan et al. (1997). The solid line denotes $\mathrm{r}_{\mathrm{m}} \mathrm{t}_{\mathrm{gen}}=1.38 \mathrm{M}^{-0.067}$, which is the best-fit to the data and has an average scatter of 0.2 dex. The best-fitted relation is in overall agreement with the predicted Eq 9, with no relevant slope with body mass.

The normalizations from the best-fit to the data in Figs $2 \& 3$, gives estimations for $\mathrm{r}_{\mathrm{m}} \mathrm{t}_{\text {life }} \sim 3.35($ Fig 2$)$ and $\mathrm{r}_{\mathrm{m}} \mathrm{t}_{\text {gen }} \sim 1.38$ (Fig 3), which in principle can be combined with Eq 8 $\& 9$, to determine some of their constants. Unfortunately, it is not possible to determine the constants $(\overline{\mathrm{b}}, \overline{\mathrm{d}})$ associated to the dimensionless birth and death rates, since Eq $8 \& 9$ are not (linearly) independent equations, but it is still possible to determine that $\frac{\mathrm{r}_{\mathrm{m}} \mathrm{t}_{\mathrm{gen}}}{\mathrm{r}_{\mathrm{m}} \mathrm{t}_{\text {life }}}=\mathrm{G} \sim 0.41$. 
This is for average lifespan in the wild because Hatton et al (2019) lifespans were normalized to those, and since maximum lifespans are about 2.5 times average ones in the wild (McCoy $\&$ Gillooly 2008), we determine from Figs $2 \& 3$ that $\frac{t_{\text {gen }}}{\mathrm{t}_{\text {life }}^{\max }}=\mathrm{G}^{\min } \sim 0.16$, which agrees with the value determined from the average of the predicted $G$ values in Table $2\left(G^{\text {min }} \sim 0.17\right)$.

\section{Summary}

In this paper, we explore the implications of the new metabolic relation (Escala 2019) for ontogenetic growth and found that it can be described by an universal ontogenetic growth curve for all studied species, without the aid of ad-hock fitting parameters. The same universal growth curve in West et al (2001) and Banavar et al (2002) is found when some dimensionless quantities are properly defined, but in our case the characteristic growth timescale $\left(t_{\text {growth }}\right)$ is set by the inverse of the heart frequency $f_{H}^{-1}$, rescaled by the ratio between the specific energies (per unit of mass) required for metabolism and to create new biomass, all quantities with clear physical or biological meaning and not obscure fitting parameters. The result of universal growth have the same interpretation as before in terms of conservation of energy, since it comes from the same Bertalanffy-type equation, but in our case the result do not rely on a specific model. Instead, this illustrates the advantages when empirical data is properly described and quantified, arising to this simple description without extra assumptions.

We also explore the implications of the discussed ontogenetic growth model for the generation time, finding that predicted $\mathrm{t}_{\text {gen }}$ can explain the origin of several 'Life History Invariants', when is it combined with the invariant number of respiration cycles per lifetime, a relation that comes from the generalization of the well known relation of constant number of heartbeats in mammal's lifetime (Levine 1997; Cook et al. 2006). In particular, the invariant ratio between lifespan and the age at maturity $\left(\mathrm{G}=\frac{\mathrm{t}_{\text {gen }}}{\mathrm{t}_{\mathrm{life}}}\right)$, which has been traditionally explained in terms of life-history evolution theory, in our formalism the value of this life- 
history invariant it is predicted in terms of the relevant energetics and the invariant number of respiration cycles per lifetime. The predicted $\mathrm{G}$ and its variation between taxonomic groups, shows both consistency with the empirically determined value (Table 2). We also showed that other life-history invariants (Charnov 1993), comes directly from the existence of the universal the ontogenetic growth curve and the invariant G.

We finally studied the predictions for population growth, finding that the invariant $G$ implies a Mathusian Parameter $\mathrm{r}_{\mathrm{m}}$, or intrinsic population growth rate, inversely proportional to both $t_{\text {gen }}$ and $t_{\text {life. }}$ We find that these inverse relations are indeed observed in nature, with no relevant slope with body mass and relatively low scatter $\left(0.5\right.$ dex for $r_{m} t_{\text {life }}$ in Fig $2 \& 0.2$ dex for $r_{m} t_{\text {gen }}$ in Fig 3$)$. The ratio $G^{\text {min }}=\frac{t_{\text {gen }}}{t_{\text {life }}^{\text {max }}}$ that can be estimated from the two best fitted relations $\left(\frac{\mathrm{r}_{\mathrm{m}} \mathrm{t}_{\text {gen }}}{\mathrm{r}_{\mathrm{m}} \mathrm{t}_{\mathrm{life}}^{\text {max }}}=\mathrm{G}^{\mathrm{min}} \sim 0.16\right)$, which is also consistent with our predicted $\mathrm{G}^{\min }(\sim 0.17$; Table 2). Also, we find relatively constant values for the total birth and deaths per capita per characteristic time ( $\bar{b}$ and $\bar{d}$ in Eq. 8), besides the Earth's diversity of life histories in living organisms, supporting the so-called 'Equally Fitness Paradigm'.

In our formalism, the allometric scaling relations for several critical timescales and rates $\left(t_{\text {life }}, t_{\text {gen }} \& r_{m}\right)$ are all coming from being proportional to $f_{H}$, therefore, the $f_{H} \propto M^{-1 / 4}$ mass scaling directly explains other well known allometries (for lifespan, generation-time and the Fenchel allometry), naturally explaining why most biological times and rates scale as $\mathrm{M}^{-1 / 4}$ and $\mathrm{M}^{1 / 4}$ (Savage et al. 2004, Burger et al. 2021). Also, the variations from $1 / 4$ scaling should be explained in terms of the variation in the $f_{H}$ mass scaling, since such quantities $\left(t_{\text {life }}, t_{\text {gen }}\right.$ and $\left.r_{m}\right)$ should have the same mass scaling as $f_{H}$, regardless if is $1 / 4$ or not, as it was the case for the metabolic rate relation (Escala 2019).

Nevertheless, our formalism is empirically motivated and do not explain why $\mathrm{f}_{\mathrm{H}}$ should scale as $\mathrm{M}^{-1 / 4}$ or with another exponent, thus it is compatible with previous/other attempts that explains the exponent for the allometric scaling of metabolism and related quantities (West et al 1997, 1999; Banavar et al 1999, 2010; Darveau et al 2002), as far as the mechanism is based on the anatomy and physiology of the circulatory system. The difference between 
both approaches, it is similar to the difference in physics between the (empirically-based and axiomatic) laws of thermodynamics and the statistical mechanics that explain them, being in this case the new metabolic rate and the invariant number of respiration cycles per lifetime the analogous to the thermodynamical laws, a synthesis that is also required towards the formulation of a general theory of biodiversity (Marquet 2017).

Finally, it is important to emphasize that our predictions were successfully tested without the aid of any free (fitting) parameters at all. The fitting procedures were done in Figs 1, $2 \& 3$ only to compare with our predictions, but not to determine any free parameter for our formalism. Moreover, the constants that defines the critical relations for metabolism $\left(\mathrm{E}_{2019}\right)$ and lifespan $\left(\mathrm{N}_{\mathrm{r}}\right)$, are successfully tested further in Fig $1\left(\mathrm{E}_{2019}\right)$ and in the G value determine by Figs $2 \& 3\left(\mathrm{E}_{2019}\right.$ and $\mathrm{N}_{\mathrm{r}}$; Eq. 6), showing that the two simple relations for metabolism and lifespan used in this paper can successfully explain a variety of complex phenomena. This resembles the reductionism seen in physics since (at least) Galileo and Newton, where simple laws accounts for multitudes of complex phenomena.

I acknowledge partial support from the Center of Excellence in Astrophysics and Associated Technologies (PFB 06) and FONDECYT \#1181663.

\section{REFERENCES}

Anderson, J.F. (1970). Metabolic rates of spiders, Comp. Biochem. Physiol., 33, 51-72

Anderson, J.F. (1974). Responses to Starvation in the Spiders Lycosa Lenta Hentz and Filistata Hibernalis (Hentz), Ecology, 55, 576-585

Banavar, J. R., Maritan, A. Rinaldo, A. (1999). Nature 399, 130-132

Banavar J.R., J. Damuth, A. Maritan, and A. Rinaldo (2002). Ontogenetic growth: modelling universality and scaling. Nature 420:626. 
Banavar JR, Moses ME, Brown JH, Damuth J, Rinaldo A, Sibly RM (2010). A general basis for quarter-power scaling in animals. Proc Natl Acad Sci USA 107:15816-15820

Basener, W. F. \& Sanford, J.C. (2018). The fundamental theorem of natural selection with mutations. J. Math. Biol. 76:1589-1622

von Bertalanffy, L. (1957). Quantitative laws in metabolism and growth. Q. Rev. Biol. 32, $217-231$

R. J. H. Beverton, S. J. Holt (1959), in Ciba Foundation Colloquia in Ageing. V. The Lifespan of Animals, G. E. W. Wolstenholme, M. O?Connor, Eds. (Churchill, London), pp. $142-177$

Bonner, J.T. (1965). Size and Cycle, Princeton University Press, USA.

Bridgman, P. W. (1922). Dimensional Analysis, Yale University Press

Brody, S. (1945). Bioenergetics and Growth. Reinhold, New York.

Brown, J.H. et al (2018). Equal fitness paradigm explained by a tradeoff between generation time and energy production rate. Nat. Ecol. Evol. 2, 262-268

Calder, W.A. III (1968) Respiratory and heart rates of birds at rest. Condor 70, 358-365

Calder, W.A. (1984). Size, Function, and Life History (Harvard Univ. Press, Cambridge, MA).

Charnov, E. L. \& Berrigan, D. (1990). Dimensionless numbers and life history evolution: age of maturity versus the adult lifespan. Evolutionary Ecology, 4, 273-275

Charnov, E. L. \& Berrigan, D. (1991). Evolution of life history parameters in animals with indeterminate growth, particularly fish. Evolutionary Ecology, 5, 63-68

Charnov, E. L. (1993). Life History Invariants: Some Explorations of Symmetry in Evolutionary Ecology (Oxford Univ. Press, Oxford). 
Charnov, E. L. (2008). Fish growth: Bertalanffy k is proportional to reproductive effort. Environ Biol Fish (2008) 83:185-187

Charnov, E. L., Turner, T. F., Winemiller, K. O. (2001). Proc. Natl. Acad. Sci. U.S.A. 98, 9460.

Clark, T. D. and Farrell, A. P. (2011). Effects of body mass on physiological and anatomical parameters of mature salmon: evidence against a universal heart rate scaling exponent. The Journal of Experimental Biology 214, 887-893

Cook, S. et al. (2006). High heart rate: a cardiovascular risk factor? European Heart Journal, $27,2387-2393$

Cushing, D.H. (1968). Fischeries biology. Univ. Wisconsin Press, Madison, Wisconsin.

Darveau CA, Suarez RK, Andrews RD, Hochachka PW (2002). Allometric cascade as a unifying principle of body mass effects on metabolism. Nature 417:166-170

Detweiler, D.K. \& Erickson, H.H. (2004). Regulation of the Heart, in Dukes' Physiology of Domestic Animals, 12th ed., Reece WO, Ed. Copyright by Cornell University.

Dillingham, P.W. et. al (2016). Improved estimation of intrinsic growth rmax for long-lived species: integrating matrix models and allometry. Publications, Agencies and Staff of the U.S. Department of Commerce. Paper 535.

Dodds, P.S., Rothman, D.H. \& Weitz, J.S., (2001). Re-examination of the 3/4-law of Metabolism, Journal of Theoretical Biology , 209, 9-27

Duncan, R.P. et al. (2007). Testing The Metabolic Theory of Ecology: Allometric Scaling Exponents in Mammals, Ecology, 88, 324-333

Escala, A. (2019). The principle of similitude in biology. Theoretical Ecology 12 (4), 415-425.

Escala, A. (2020). Universal Relation for Life-span Energy Consumption in Living Organisms: Insights for the origin of aging. bioRxiv 2020.04.07.030528 
Fenchel, T. (1974). Intrinsic Rate of Natural Increase: The Relationship with Body Size, Oecologia, 14, 317-326

Fisher, R.A. (1930) The genetical theory of natural selection. Oxford: Clarendon Press, New York: Dover

Gardner, A., Allsop, D. J., Charnov, E. L., West, S. A. (2005). Am. Nat. 165, 551.

Gillooly, J.F., Brown, J.H., West, G.B., Savage, V.M. \& Charnov, E.L. (2001). Effects of size and temperature on metabolic rate, Science, 293, 2248-2251.

Harris, C. (2009). A Guide to Traditional Pig Keeping. Good Life Press.

Hatton, I.A. et al. (2019). Linking scaling laws across eukaryotes, PNAS, 116 (43) 2161621622

Hou C., W.Y. Zuo, M.E. Moses, W.H. Woodruff, J.H. Brown, and G.B. West (2008). Energy uptake and allocation during ontogeny. Science 322:736-739.

Jurgens, K.D. et al. (1996). Heart and respiratory rates and their significance for convective oxygen transport rates in the smallest mammal, the Etruscan shrew Suncus etruscus. The Journal of Experimental Biology 199, 2579-2584

Kleiber, M. (1932). Body size and metabolism, Hilgardia, 6, 315-351

Kovacs L, Kezer FL, Tozser J, Szenci O, Poti P, Pajor F (2015) Heart Rate and Heart Rate Variability in Dairy Cows with Different Temperament and Behavioural Reactivity to Humans. PLoS ONE 10(8): e0136294

Malthus, T. R. (1798). An Essay on the Principle of Population. J. Johnson, London

Marquet, PA. (2017). Integrating macroecology through a statistical mechanics of adaptive matter. Proc Natl Acad Sci;114(40):10523-10525. 
Mahida, N., \&is Aohagi, Y. (2001). Electrocardiography, Heart Rates, and Heart Weights of Free-Living Birds. Journal of Zoo and Wildlife Medicine, Vol. 32, No. 1, pp. 47-54

Moses, M. E. et al. (2008). "Revisiting a Model of Ontogenetic Growth: Estimating Model Parameters from Theory and Data," American Naturalist, vol. 171, no. 5, pp. 632-645

Pauly, D. (1980). On the interrelationships between natural mortality, growth parameters and mean environmental temperature in 175 fish stocks. J Cons Int Explor Mer 39:175-192

Price GR (1972) Fisher's fundamental theorem made clear. Ann Hum Genet Lond 36:129-140

Ramsey, J. J., Harper, M. E. and Weindruch, R. (2000). Restriction of energy intake, energy expenditure, and aging. Free Rad. Biol. Med. 29, 946-968.

Reiss M.J. (1989). The allometry of growth and reproduction. Cambridge University Press, Cambridge.

Ricklefs R.E. (2003). Is rate of ontogenetic growth constrained by resource supply or tissue growth potential? a comment on West et al.'s model. Funct Ecol 17:384-393.

Savage, V. M., J. F. Gillooly, W. H. Woodruff, G. B. West, A. P. Allen, B. J. Enquist, and J. H. Brown (2004). The predominance of quarter-power scaling in biology. Functional Ecology 18:257-282.

Schmidt-Nielsen, K. (1984). Scaling: why is animal size so important?, Cambridge University Press.

Speakman, J.R. (2005). Body size, energy metabolism and lifespan. The Journal of Experimental Biology 208, 1717-1730.

Wardle, C. S. , \& Kanwisher, J. W. (1973). The significance of heart rate in free swimming cod, Gadus morhua: Some observations with ultra?sonic tags, Marine Behaviour and Physiology, 2:1-4, 311-324 
Weibel, E.R., Bacigalupe, L.D., Schmidt, B. \& Hoppeler, H. (2004). Allometric scaling of maximal metabolic rate in mammals: muscle aerobic capacity as a determinant factor. Respiration Physiology and Neurobiology, 140, 115-132

Welty, J.C., \& Baptista, L. (1988). The Life of Birds. Saunders College Publishing, New York.

West, G.B., Brown, J.H. \& Enquist, B.J. (1997). A general model for the origin of allometric scaling laws in biology. Science 276, 122-126

West G, Brown J, Enquist BJ (1999). The fourth dimension of life: fractal geometry and allometric scaling of organisms. Science 284:1677-1679

West, G.B.,Brown, J.H. \& Enquist, B.J. (2001) Nature 413, 628-631.

West, G., Brown, J. (2004). Life's Universal Scaling Laws, Physics Today, 57, 36-42

White, Craig R. et al. (2007). Allometric Exponents Do Not Support a Universal Metabolic Allometry, Ecology, 88, 315-323 